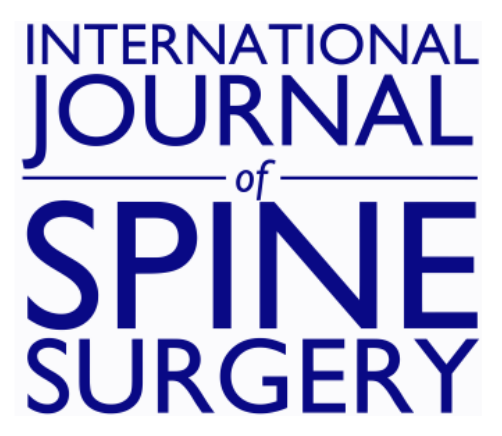

\title{
Cardiac Cement Embolism After Thoracic Kyphoplasty: Successful Conservative Treatment With 4-Year Follow-Up
}

Sarah Mills, Javier Pizones, Luis Rodrigo Merino Rueda, Gloria Talavera, José Miguel Sánchez-Márquez and Nicomedes Fernández-Baíllo

Int J Spine Surg published online 17 February 2022

http://ijssurgery.com/content/early/2022/02/17/8173

This information is current as of April 26, 2023.

Email Alerts Receive free email-alerts when new articles cite this article. Sign up at: http://ijssurgery.com/alerts 


\title{
Cardiac Cement Embolism After Thoracic Kyphoplasty: Successful Conservative Treatment With 4-Year Follow-Up
}

\author{
SARAH MILLS, MD ${ }^{1}$; JAVIER PIZONES, MD, PHD ${ }^{1}$; LUIS RODRIGO MERINO RUEDA, MD ${ }^{1}$; GLORIA TALAVERA, \\ MD $^{1}$; JOSÉ MIGUEL SÁNCHEZ-MÁRQUEZ, MD, PHD ${ }^{1}$; AND NICOMEDES FERNÁNDEZ-BAÍLLO, MD ${ }^{1}$ \\ ${ }^{I}$ Spine Unit, Department of Orthopaedic Surgery, La Paz Hospital Universitario, Madrid, Spain
}

\begin{abstract}
Background: The present case report describes a complication after a percutaneous spine surgery technique that is highly uncommon in clinical practice: a bone cement cardiac embolism. This rare complication emphasizes the importance of this case, which is also interesting considering the midterm follow-up. Documented cardiac embolisms published in the literature (which are scarce) describe the acute phase of these cases but lack follow-up. There are no systematic reviews on this topic, only caseby-case presentations, and surgeons are not aware of its real implications.

Case: We report a case of an 84-year-old man who developed sudden thoracic and spinal pain associated with $82 \%$ saturation and dyspnea a few hours after 4-level thoracic spine vertebroplasty and kyphoplasty. Imaging revealed multiple bone cement embolisms in his lung and heart. Because the patient was hemodynamically stable, cardiologists recommended conservative treatment with low molecular weight heparin, without embolus removal. At 4-year follow-up, the patient remained asymptomatic.

Conclusion: Cardiac cement embolization following percutaneous techniques represents a life-threatening situation that should be ruled out if the patient presents symptoms during the early postoperative period. Treatment may vary from conservative to emergency open-heart surgery.
\end{abstract}

Case Report

Keywords: vertebroplasty, kyphoplasty, cement cardiac embolism, vertebral fractures, osteoporotic fractures, complications

\section{INTRODUCTION}

Nowadays, percutaneous surgical techniques to treat osteoporotic vertebral compression fractures (OVCFs) are common procedures of our daily practice. However, the injection of bone cement can have related complications. The most frequent is the leakage of cement into the vertebral body surroundings or the venous system with ulterior migration to the pulmonary arterial tree.

The incidence of cement leakage has been demonstrated to be higher in vertebroplasties than in kyphoplasties. ${ }^{1}$ In most cases, this complication has no clinical relevance, but symptoms, when present, can be devastating. ${ }^{2}$ One uncommon secondary leakage complication is cement migration to the cardiac system. Few studies have reported this rare complication. Information about its clinical manifestations, diagnostic steps to undertake, and treatment options are scarce and still subject to debate.

We report a case of a patient who suffered acute chest pain and dyspnea after multilevel thoracic vertebroplasty and kyphoplasty due to OVCFs. After a thorough examination, in addition to the pulmonary leakage, a right ventricle cement embolization was detected. The aim of this report is to present the clinical features of the case and perform a literature review to describe the sequence of examinations and the treatment decision-making process after a cement cardiac embolism.

\section{CASE REPORT}

An 84-year-old man presented at our clinic complaining of spinal pain without previous trauma. The pain started 3 weeks prior, was located at the thoracolumbar junction with no radiating features, and improved with bed rest.

Upon physical examination, no deformities were observed. The pain was located at the midthoracic spine and limited the patient's range of movement. The neurological examination was normal, deep tendon reflexes were normal, and there was no loss of sphincter control.

Thoracolumbar anteroposterior and lateral radiographs showed anterior wedging of the T9 and T11 vertebrae. Magnetic resonance imaging revealed acute OVCFs at T9 and T11 levels (Figure 1). 
As the symptoms did not improve despite conservative treatment, we proposed a surgical percutaneous cement injection procedure to relieve the pain. T9 and T11 kyphoplasties with adjacent T8 and T10 preventive vertebroplasties were performed. A polymethyl methacrylate-based acrylic bone cement containing radiopaque zirconium dioxide (BonOs cement) was employed. We injected $3 \mathrm{cc}$ per level in kyphoplasties, which were performed first, and $2 \mathrm{cc}$ per level in vertebroplasties. This is a high-viscosity cement based on methyl-methacrylate, which also contains benzoyl peroxide, dimethyl-p-toluidine, and hydroquinone. The procedure was developed under continuous fluoroscopy control. We did not perform a venogram before cement injection as it is not a common technique at our institution. No anterior leakage was observed intraoperatively, and no hemodynamic changes occurred throughout the procedure.

Six hours after the surgery, the patient began to have acute spinal and chest pain and shortness of breath. The oximeter measured $82 \%$ oxygen saturation. Blood pressure and heart rate were normal.

Thoracic and lumbar radiographs to rule out potential new acute adjacent fractures were obtained. These images revealed radiopaque bodies in both lungs (Figures 2 and 3). Blood analysis was performed with no abnormalities except for an increase in D-dimer level $(6347 \mathrm{ng} / \mathrm{mL})$. Electrocardiogram (ECG) did not reveal any pathological findings. A pulmonary embolism was the main suspicion, despite heart rate and blood pressure being normal. Pulmonary vascular computed tomography (CT) images revealed multiple bone cement embolisms in his right lung and an unsuspected cement embolism inside his right ventricle apex $(20 \times 4$ $\mathrm{mm}$ ) (Figure 4).

Cardiologists evaluated the patient, and after the physical examination, they decided to perform echocardiography. The cement embolism was seen as a highly echogenic fiber-like structure deeply hosted in his ventricular septal partition (Figure 5).

As the patient was hemodynamically stable and the embolus did not move with ventricular contractions, the cardiologists recommended not removing the cement because of the high risk of muscular damage and potential secondary hemodynamic instability.

Treatment began with oxygen therapy and low molecular weight heparin (LMWH) $-80 \mathrm{mg}$ of enoxaparin every 12 hours. Over the following days, our patient suffered a huge spinal subcutaneous hematoma that required blood transfusions (4 units) and an LMWH dose reduction for a few days. An exploratory angiography was performed and no arterial lesions were found. Therefore, embolization was not required.

Initial spinal pain persisted for 3-4 days and finally disappeared after the administration of intravenous painkillers. Once the hematoma was under control, the patient was discharged without oxygen requirements. Following the cardiologist's advice, the patient continued with LMWH for 16 months. Finally, treatment was
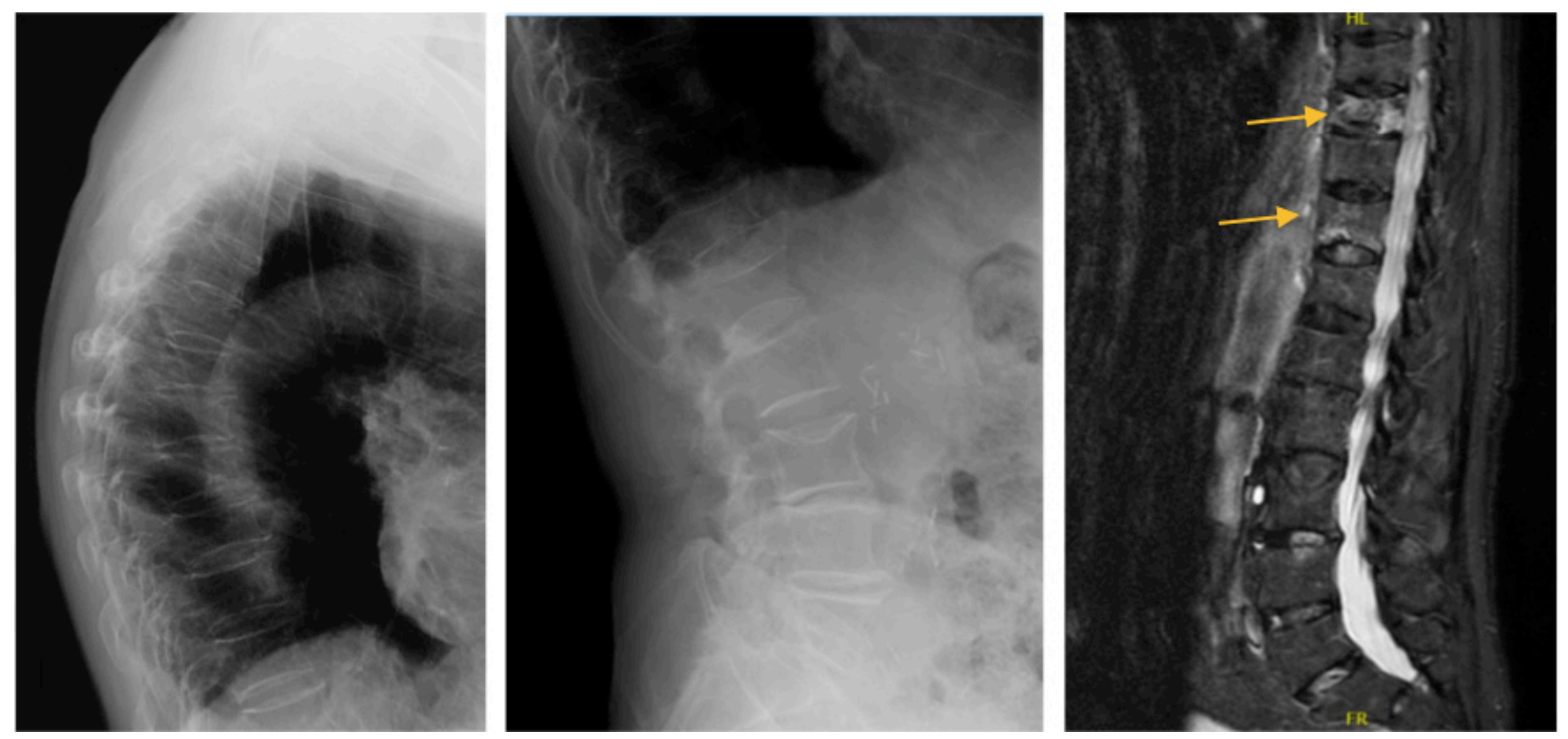

Figure 1. Preoperative images consisting of spinal anteroposterior and lateral $\mathrm{x}$-ray images and T2 short tau inversion recovery sagittal magnetic resonance image, where multiple osteoporotic fractures can be seen (some of which are chronic lesions) with acute lesions in T9 and T11 levels (marked with yellow arrows). 


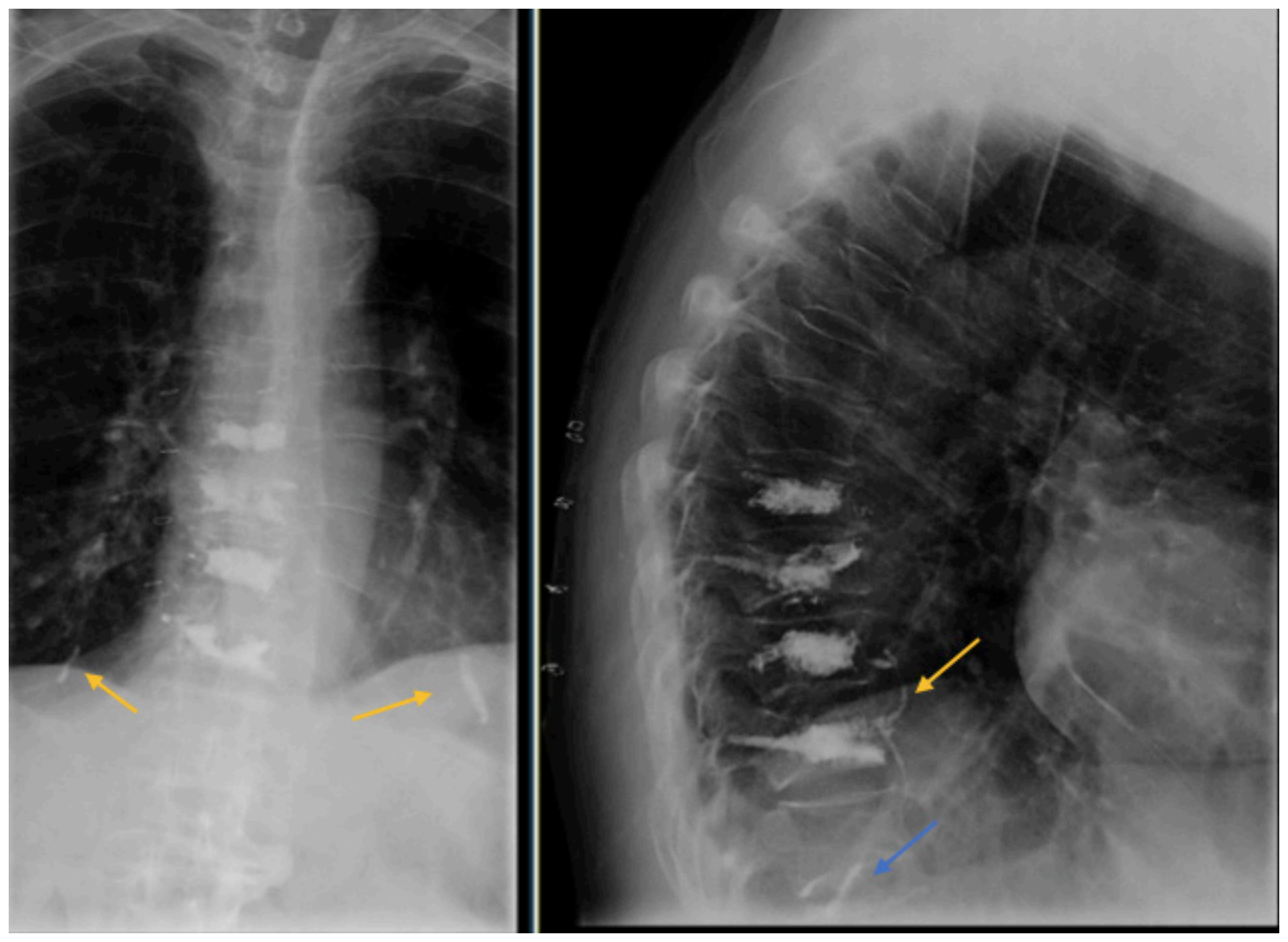

Figure 2. Postoperative anteroposterior and lateral $\mathrm{x}$-ray images of the spinal region, showing results of kyphoplasty in T9 and T11 and vertebroplasty in T8 and T10 levels. Cement leakage is observed anterior to the vertebral bodies (yellow arrow) and inferior in the lung (blue arrow). In the anteroposterior view, a cylinder can be seen in the right lung with another one over the heart silhouette (marked with yellow arrows).

changed to oral anticoagulant therapy (apixaban) due to an iliac pseudoaneurysm thrombosis.

Four years after the procedure, the patient remains asymptomatic and continues with his daily activities. He is still on anticoagulant therapy at home and continues to make scheduled visits to our clinic.

\section{DISCUSSION}

OVCFs are becoming a major healthcare concern, with their incidence increasing rapidly. ${ }^{3}$ Percutaneous minimally invasive surgical techniques, such as vertebroplasties and kyphoplasties, are common procedures for the treatment of vertebral fractures including osteoporotic, pathologic, traumatic, or tumor lesions. Although uncommon, described complications of these procedures are hemorrhage, infection, neural injuries, cement leakage, and fractures of the adjacent vertebrae. ${ }^{4}$

These techniques consist of a cement (usually polymethyl methacrylate or biodegradable calcium phosphate) injection inside the vertebral body through a percutaneous transpedicular approach (with the previous insertion of an expansive balloon in the case of kyphoplasty) to strengthen and stabilize the fracture and relieve the pain. Cement injection can result in leakage into the spinal canal or discs and extravasation into the external vertebral venous plexus. Venous leakage initiates at the segmental and/or paravertebral veins and can then travel through the azygos or hemiazygos system or directly through the inferior vena cava, reaching the lungs and causing pulmonary cement embolism. Cement could be the reason for a pulmonary embolism itself or by the displacement of fat or bone marrow through the venous system. ${ }^{5}$ Venogram before cement injection is not a common technique at our institution, despite some colleagues recommend it as a safe procedure. $^{6}$

According to the literature, the cement extravasation rate varies widely, from $5 \%$ to more than $80 \%$. 


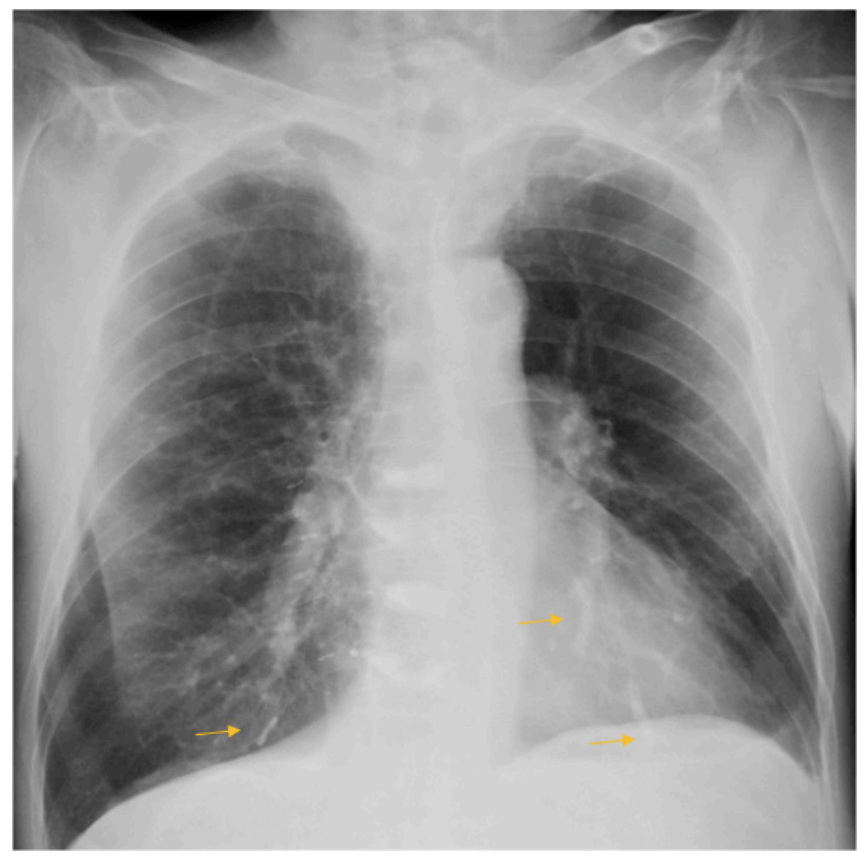

Figure 3. Postoperative anteroposterior chest $\mathrm{x}$-ray image, showing cement cylinders over the heart silhouette and in the base of the right lung (marked with yellow arrows).

However, the majority of the cases are asymptomatic. When using a CT to detect the presence of cement in the pulmonary arterial system, this rate rises to $63 \%-87 \% .^{3}$
In rare cases, death can occur quite suddenly after a massive pulmonary cement embolism or in the event of cardiac structural lesions. ${ }^{7}$

Very few reports can be found in the literature regarding cardiac cement migration., ${ }^{2,8-12}$ Our review showed an age range from 55 to 80 years. Frequent clinical manifestations are thoracic pain, heart failure, or palpitations. The most common secondary diagnostic examinations used are ECG, echocardiography, and CT. Conservative treatment was selected in 2 cases, ${ }^{8,12}$ but it was effective only in 1 ; one patient presented with a ventricular perforation caused by the cement itself and required emergency open-heart surgery. ${ }^{12}$ Cement removal by endovascular techniques was the chosen procedure in 2 cases $^{9,11}$ achieving satisfactory results. One patient required open-heart surgery, ${ }^{10}$ and one patient died suddenly before any treatment could be performed. $^{2}$

If a patient begins to show symptoms of chest pain and dyspnea shortly after a percutaneous procedure, we should remember that radiographs have limited sensitivity for the diagnosis. Therefore, if a cardiac embolism is suspected, chest CT and echocardiography are the diagnostic examinations to be performed. ${ }^{10}$

In case of such complications, therapeutic strategies may vary depending on the patient's status. Conservative treatment (oxygen support and anticoagulation
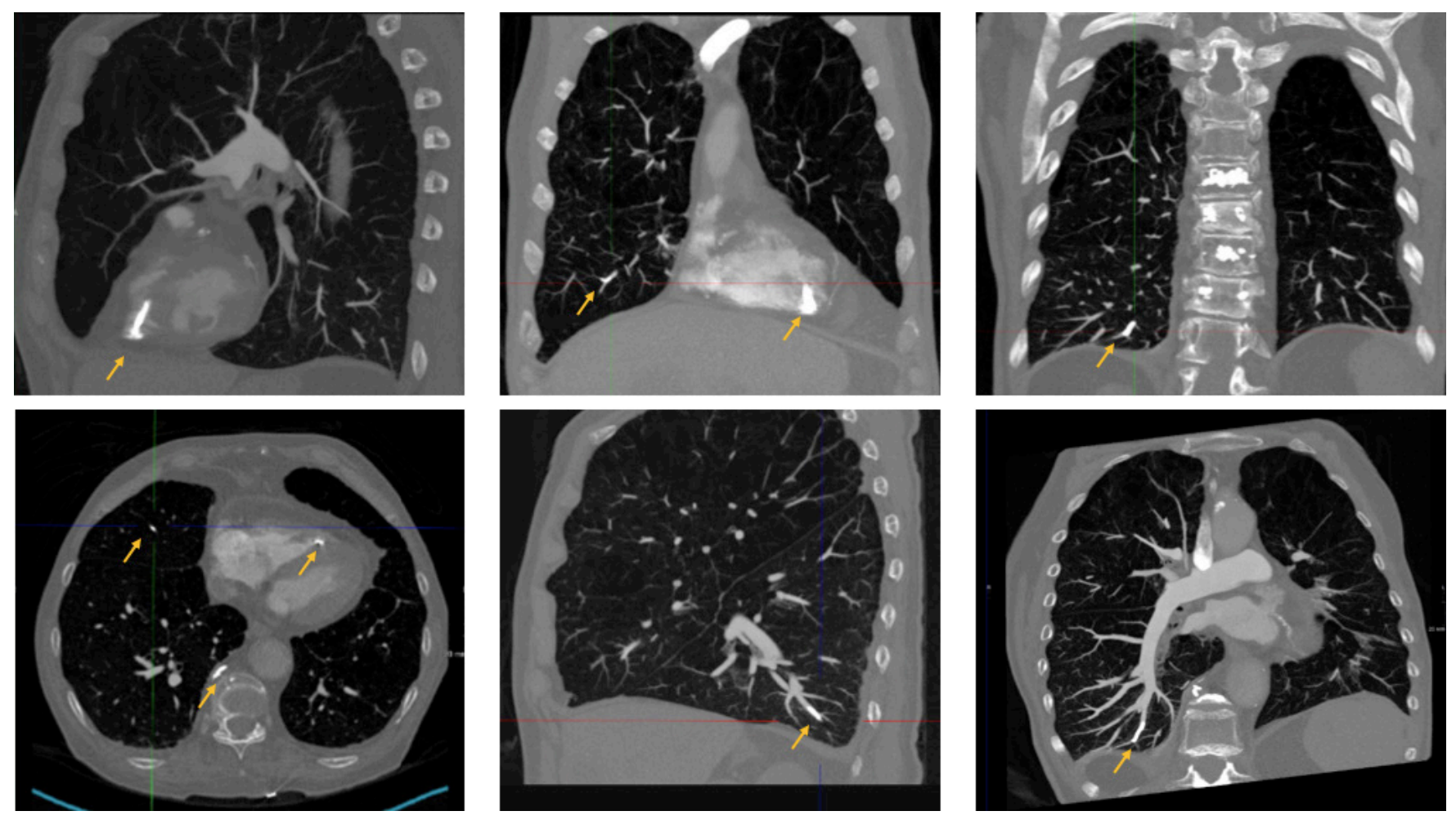

Figure 4. Postoperative computed tomography pulmonary vascular images showing multiple cement embolus (marked with yellow arrows). 

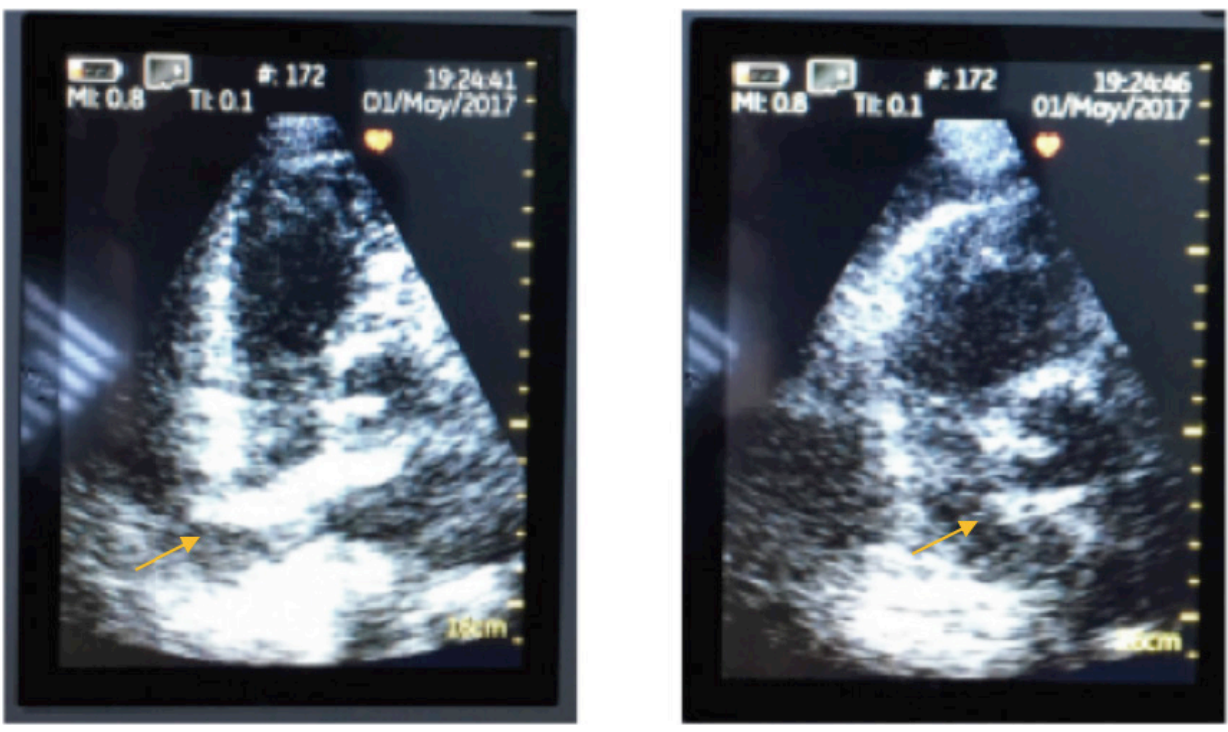

Figure 5. Postoperative echocardiogram locates the cement embolism in the ventricular septal partition (marked with yellow arrows).

therapy) can be effective, but patients must be selected carefully. This approach can be applied to hemodynamically stable patients with an immobile embolus. This approach seeks to promote foreign body endothelization inside the cardiac cavity to avoid further cement embolization.

In cases of hemodynamic instability or embolus intracardiac mobility, the removal of the embolus seems to be necessary. Endovascular techniques, when possible, can be helpful with fewer risks compared to open-heart surgery. If the embolus cannot be reached through an endovascular approach, or if the heart presents a structural injury, open-heart surgery is required through a median sternotomy and extracorporeal circulatory support. These procedures carry a high risk of fatal complications. ${ }^{3}$

Risk factors for cement extravasation can be the insufficient polymerization of the cement, cement type and viscosity, injected amounts (as leakage is dose dependent), or the number of levels augmented. ${ }^{1}$ Highviscosity cement is strongly recommended; the proper setting time of the cement should be respected (and this might have been the failure in this case); and frequent fluoroscopy control during the filling should be performed. $^{3,7}$

Some patients develop symptoms weeks or years after the procedure, which is why some authors suggest routine chest radiography, echocardiography, and chest CT after percutaneous surgical procedures, to find asymptomatic embolisms and avoid subsequent clinical complications. ${ }^{8}$

\section{CONCLUSION}

Cardiac embolisms are uncommon complications after percutaneous cement injection for OVCFs. They are usually symptomatic, unlike pulmonary embolisms. Symptoms can appear immediately after cement injection, or they can appear weeks and even months later. Typical clinical manifestations are palpitations, thoracic/chest pain, or heart failure. When suspected, ECG, echocardiography, and CT are the recommended examinations. The treatment may vary from conservative, with oxygen and anticoagulant therapy, to endovascular or open techniques to remove the embolus depending on the patient's hemodynamic stability or embolus mobility.

\section{REFERENCES}

1. Hulme PA, Krebs J, Ferguson SJ, Berlemann U. Vertebroplasty and kyphoplasty: a systematic review of 69 clinical studies. Spine. 2006;31(17):1983-2001. doi:10.1097/01.brs.0000229254. $89952.6 b$

2. D'Errico S, Niballi S, Bonuccelli D. Fatal cardiac perforation and pulmonary embolism of leaked cement after percutaneous vertebroplasty. J Forensic Leg Med. 2019;63:48-51. doi:10.1016/j. jflm.2019.03.004

3. Hsieh M-K, Kao F-C, Chiu P-Y, et al. Risk factors of neurological deficit and pulmonary cement embolism after percutaneous vertebroplasty. J Orthop Surg Res. 2019;14(1):406. doi:10.1186/ s13018-019-1459-4

4. Filippiadis DK, Marcia S, Masala S, Deschamps F, Kelekis A. Percutaneous vertebroplasty and kyphoplasty: current status, new developments and old controversies. Cardiovasc Intervent Radiol. 2017;40(12):1815-1823. doi:10.1007/s00270-017-1779-x

5. Iwanaga J, Rustagi T, Ishak B, et al. Venous drainage of lumbar vertebral bodies: anatomic study with application to kyphoplasty, 
vertebroplasty, and pedicle screw complications. World Neurosurg. 2020;137:e286-e290. doi:10.1016/j.wneu.2020.01.174

6. Vasconcelos C, Gailloud P, Beauchamp NJ, Heck DV, Murphy KJ. Is percutaneous vertebroplasty without pretreatment venography safe? Evaluation of 205 consecutives procedures. AJNR Am J Neuroradiol. 2002;23(6):913-917.

7. Monticelli F, Meyer HJ, Tutsch-Bauer E. Fatal pulmonary cement embolism following percutaneous vertebroplasty (PVP). Forensic Sci Int. 2005;149(1):35-38. doi:10.1016/j.forsciint.2004.06.010

8. Cianciulli TF, Mc Loughlin DE, Morita LA, Saccheri MC, Lax JA. Bone cement cardiac and pulmonary embolism. Echocardiography. 2017;34(8):1239-1241. doi:10.1111/echo.13580

9. Park JS, Kim J, Lee Y, Gwon JG, Park YS. Intra-cardiac embolism of a large bone cement material after percutaneous vertebroplasty removed through a combination of an endovascular procedure and an inferior vena cava exploration: a case report. J Korean Med Sci. 2018;33(19):19. doi:10.3346/jkms.2018.33.e141

10. Song Y, Huang X, Wu L. Removal of intracardiac bone cement embolism after percutaneous kyphoplasty: a case report. Medicine (Baltimore). 2020;99(11):e19354. doi:10.1097/ MD.0000000000019354

11. Shamekhi J, Düsing P, Sedaghat A, Kütting D, Nickenig G, Sinning JM. Healing a heart of stone: percutaneous extraction of cardiopulmonary cement embolism. JACC Cardiovasc Interv. 2020;13(4):532-533. doi:10.1016/j.jcin.2019.09.044

12. Swojanowsky P, Brinkmeier-Theofanopoulou M, Schmitt C, Mehlhorn U. A rare cause of pericardial effusion due to intracardiac cement embolism. Eur Heart J. 2018;39(32):32. doi:10.1093/ eurheartj/ehy208
Funding: The author(s) received no financial support for the research, authorship, and/or publication of this article.

Declaration of Conflicting Interests: The authors report no conflicts of interest in this work.

Patient consent for publication: The patient was informed that the case would be submitted for publication, and he provided consent.

Ethics Approval: This study has IRB approval/ Research Ethics Committee.

Corresponding Author: Sarah Mills, Spine Unit, Department of Orthopaedic Surgery, Hospital Universitario La Paz, Madrid, Spain; sarah.mills@ salud.madrid.org

Published 15 February 2022

This manuscript is generously published free of charge by ISASS, the International Society for the Advancement of Spine Surgery. Copyright (C) 2022 ISASS. To see more or order reprints or permissions, see http:// ijssurgery.com. 